\title{
Analyse the electrical characteristics of an MagnetoHydroDynamic generator for maximizing the thermal efficiency
}

- Le Chi Kien

Ho Chi Minh City University of Technology and Education

(Manuscript Received on February 10 ${ }^{\text {th }}$, 2014; Manuscript Revised August 13 ${ }^{\text {th }}, 2014$ )

\section{ABSTRACT:}

In this study, a Faraday type MagnetoHydroDynamic (MHD) generator is studied to consider the effect of electrical characteristics to the thermal efficiency. The generator performance is specified by optimizing the cycle efficiency with respect to the load parameter and by optimizing output power density with respect to seed fraction and operating pressure. As the calculation results, the value of load parameter, which

Keywords: MHD generator, thermal efficiency, electrical characteristic, load parameter, output power density.

\section{INTRODUCTION}

Techniques of Magnetohydrodynamic (MHD) power generation are being studied with increasing interest for the development of high temperature materials and high field strength magnets progresses. Devices using these techniques are to take the place of the turbo generator in a conventional power generation cycle. Several studies have been proposed that combine Rankine, Brayton, or hybrid cycles with liquids, vapors, and mixtures of these two as proposed working fluids [1-4]. Some of these studies may be used in a Brayton cycle where the working fluid is an alkali metal vapor seeded in a noble gas. These studies utilize the induced electric field of the plasma to increase the electron temperature. Each of these studies considers a particular noble gas and seed for which high conductivity was attained. In these studies, however, no attempt has been made to consider the effect of electrical characteristics such as load parameter, electrical conductivity of MHD generator for a specified generator operating under conditions appropriate for maximizing the thermal efficiency.

In this study a constant area linear duct with segmented electrodes operating as a Faraday type MHD generator is studied to consider the effect of electrical characteristics to the thermal efficiency. The magnetic field is constant and unaffected by the fluid. The current through each pair of electrodes is adjusted so that the 
generated voltage is constant. The working fluid is a noble gas seeded with cesium, and the effects of viscosity and heat conduction are neglected. The comparison between different seeded noble gas working fluids will be examined for the optimum conditions to be obtained.

\section{THERMODYNAMIC CYCLE EFIFICIENCY}

A Brayton cycle is considered with temperatures defined as shown in figure 1. The compressor efficiency, generator (isentropic) efficiency, and the generator efficiency are defined as follows:

$$
\begin{aligned}
& T_{2}-T_{1}=\eta_{\text {comp }}\left(T_{2^{\prime}}-T_{1}\right) \\
& \eta_{S}\left(T_{4}-T_{5}\right)=T_{4}-T_{5^{\prime}} \\
& T_{3}-T_{2^{\prime}}=T_{5^{\prime}}-T_{6}=\eta_{\text {reg }}\left(T_{5^{\prime}}-T_{2^{\prime}}\right)
\end{aligned}
$$

where the primed subscripts denote actual state points in figure 1. It is of interest to relate the generator efficiency to the variables defined in the text and to discuss some of the implications of the concept. The efficiency $\eta_{S}$ can be expressed in terms of the solution to the generator equations as follows: From the definition of $\eta_{s}$ :

$\eta_{S}=\frac{T_{4}-T_{5^{\prime}}}{T_{4}-T_{5}}=\frac{\eta_{c o n v}}{1-\frac{T_{5}}{T_{4}}}=\frac{\eta_{c o n v}}{1-Y}$

However, $Y=\left(T_{5} / T_{4}\right)=\left(p_{L} / p_{H}\right)^{(\gamma-1) / \gamma}$ must also be expressed in terms of the generator variables. The ratio of total pressures $p_{L} / p_{H}$ is expressed in terms of the dimensionless exit static pressure $P$, the exit gas velocity $U$, and the total temperature ratio $T_{5} / T_{4}$ :

$$
\frac{p_{L}}{p_{H}}=\frac{P \gamma M_{0}^{2}}{\left(P \gamma M_{0}^{2} U\right)^{\gamma /(\gamma-1)}}\left(\frac{T_{5^{\prime}}}{T_{4}}\right)^{\gamma /(\gamma-1)}
$$

so that

$$
\eta_{S}=\frac{\eta_{c o n v}}{1-\frac{1-\eta_{c o n v}}{U\left(\gamma M_{0}^{2} P\right)^{1 / \gamma}}}
$$

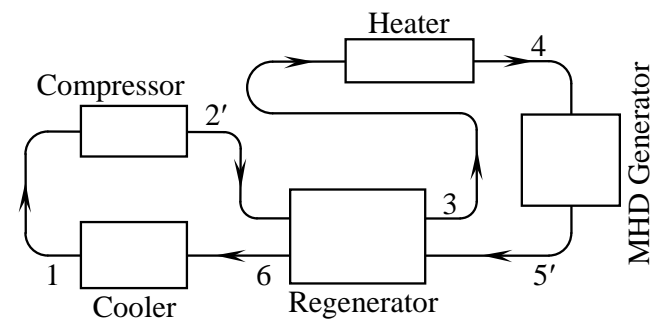

(a) Schematic diagram

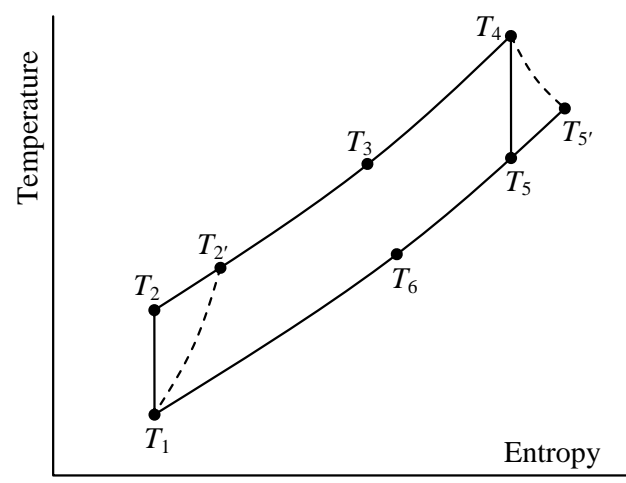

(b) Temperature-entropy diagram

Figure 1. Brayton cycle temperature definitions

It should be noted that this isentropic efficiency is based on total properties. An isentropic change in total enthalpy that is not zero can occur if the work is being done. This can be illustrated as follows.

The momentum and energy equations of the MHD generator are

$$
\begin{aligned}
& \rho u \frac{\mathrm{d} u}{\mathrm{~d} x}+\frac{\mathrm{d} p}{\mathrm{~d} x}+j B=0 \\
& \rho u \frac{\mathrm{d} h}{\mathrm{~d} x}+\rho u^{2} \frac{\mathrm{d} u}{\mathrm{~d} x}-j E_{\perp}=0
\end{aligned}
$$

Multiplying equation (7) by $u$ and subtracting from the equation (8) yield

$$
\rho u\left(\frac{\mathrm{d} h}{\mathrm{~d} x}-\frac{1}{\rho} \frac{\mathrm{d} p}{\mathrm{~d} x}\right)=j\left(E_{\perp}+u B\right)=\frac{j^{2}}{\sigma}
$$

\section{Trang 38}


From the Second Law of Thermodynamics, however, the left side of this equation can be written as

$\rho u T \frac{\mathrm{d} s}{\mathrm{~d} x}=\frac{j^{2}}{\sigma}$

so that a constant entropy process can occur if $\sigma$ approaches infinity. Hence, for an MHD generator, the isentropic efficiency compares the actual generator to a generator using an infinitely conducting working fluid.

The parameters $Y$ and $Z(Y, Z \leq 1)$ are defined as

$$
Y=\left(\frac{p_{L}}{p_{H}}\right)^{(\gamma-1) / \gamma}, Z=\frac{T_{2}}{T_{4}}
$$

The thermodynamic efficiency for zero pressure drops through the heater, regenerator, and cooler may be expressed in terms of these parameters as

$$
\eta_{\text {th }}=\frac{\left(\eta_{S} \eta_{\text {comp }}-Z\right)(1-Y)}{\eta_{\text {comp }}-\left\{\begin{array}{l}
Z\left(1-\eta_{\text {reg }}\right)\left[1-\left(1-\eta_{\text {comp }}\right) Y\right]+ \\
+\eta_{\text {reg }} \eta_{\text {comp }}\left[1-(1-Y) \eta_{S}\right]
\end{array}\right\}}
$$

If the cycle is to be used in the space environment, then it is desirable to minimize radiator area. The temperature ratio $Z$, which minimizes the area, can now be determined. The heat radiated per unit electric power developed can be expressed as

$$
\frac{Q_{\text {rad }}}{W_{\text {th }}}=\frac{1-\eta_{\text {th }}}{\eta_{t h}}
$$

where $W_{t h}$ is thermodynamic work delivered by cycle,

$Q_{\text {rad }}=\varepsilon_{\text {eff }} \sigma_{S B} A_{r} T_{\text {ave }}^{4}$

and

$$
T_{\text {ave }}^{4}=\frac{3 T_{6}^{3} T_{1}^{3}}{T_{6}^{2}+T_{6} T_{1}+T_{1}^{2}}
$$

The area $A_{r}$ (radiator area), required for a fixed maximum temperature $\mathrm{T}_{4}$ can be obtained from

$$
\frac{\varepsilon_{e f f} \sigma_{S B} A_{r} T_{4}}{W_{t h}}=\frac{1-\eta_{t h}}{\eta_{t h}}\left(\frac{T_{4}}{T_{\text {ave }}}\right)^{4}
$$

where $\varepsilon_{\text {eff }}$ is effective emissivity of radiator, $\sigma_{S B}$ is the Stefan-Boltzmann constant. Equation (15) is rewritten in terms of

$$
\left.\begin{array}{l}
a=\left(1-\eta_{\text {reg }}\right)\left[1-(1-Y) \eta_{S}\right] \\
b=\frac{\eta_{\text {reg }}}{\eta_{\text {comp }}}\left[Y \eta_{\text {comp }}+(1-Y)\right]
\end{array}\right\}
$$

by using equation (15) to evaluate $\left(\mathrm{T}_{4} / \mathrm{T}_{\mathrm{ave}}\right)^{4}$ and equation (2) to eliminate the temperature terms. Here, $a, b$ are machine efficiency parameters, then the area per unit power output becomes

$$
\begin{aligned}
& \frac{\varepsilon_{e f f} \sigma_{S B} A_{r} T_{4}^{4}}{W_{t h}}=\frac{1-\eta_{t h}}{3 \eta_{t h}} \frac{1}{a+(b-Y) Z} . \\
& {\left[\frac{1}{Y^{3} Z^{3}}-\frac{1}{(a+b Z)^{3}}\right]}
\end{aligned}
$$

Differentiation with respect to $Z$ produces the following equation for $Z$, which minimizes $A_{r}$, in equation (15):

$$
Z\left[4-\frac{a Y^{3} Z^{3}}{(a+b Z)^{4}-b Y^{3} Z^{4}}\right]=3 \eta_{S} \eta_{c o m p}
$$

The solution to this fifth-degree polynomial can be obtained in two special cases. The parameters $\omega$ and $v$ are defined as

$$
\left.\begin{array}{c}
\omega=\frac{Y Z}{1-(1-Y) \eta_{S}} \\
v=\frac{3 Y \eta_{S} \eta_{c o m p}}{1-(1-Y) \eta_{S}}
\end{array}\right\}
$$

Equation (16) then becomes 


$$
\omega\left[4-\frac{\left(1-\eta_{\text {reg }}\right) \omega^{3}}{\left(1-\eta_{\text {reg }}+\frac{b}{Y} \omega\right)^{4}-\frac{b}{Y} \omega^{4}}\right]=v
$$

It may be seen that when $\eta_{r e g}=1$

$$
\omega=\frac{v}{4}
$$

and when $\eta_{r e g}=0$ (and $\left.\mathrm{b}=0\right)$,

$$
\omega\left(4-\omega^{3}\right)=v
$$

These two solutions, which are plotted in figure 2 , are nearly the same for $v \leq 2$. As a matter of fact, there is a condition for which the solutions will all be the same, namely, when the second term in the brackets of the equation (16) is small compared to 4 . It can be shown that if

$\eta_{S} \geq \eta_{\text {conv }}+\frac{1-\eta_{\text {reg }}-\eta_{\text {conv }}}{\eta_{\text {comp }}\left(1+\eta_{\text {reg }}\right)}$

where $\eta_{S}(1-Y)=\eta_{\text {conv }}$

then the second term will be less than 0.4. If $\eta_{\text {reg }}=1$, the inequality is always true. For the remainder of the analysis, it will be assumed that the parameters are chosen such that this inequality is satisfied. Then, the value of $Z$ that minimizes $A_{r}$ is

$$
Z=\frac{3}{4} \eta_{S} \eta_{\text {comp }}
$$

and the thermodynamic cycle efficiency may be written as

$$
\begin{aligned}
\eta_{\text {th }}= & \frac{\frac{1}{4} \eta_{\text {conv }}}{\eta_{\text {reg }} \eta_{\text {conv }}+\left(1-\eta_{\text {reg }}\right) .} \\
& {\left[1-\frac{3}{4} \eta_{\text {conv }}\left(1+\eta_{\text {comp }} \frac{Y}{1-Y}\right)\right] }
\end{aligned}
$$

$$
\begin{aligned}
& \rho u \frac{\mathrm{d} u}{\mathrm{~d} x}+\frac{\mathrm{d} p}{\mathrm{~d} x}+j B=0 \\
& \rho u \frac{\mathrm{d} h}{\mathrm{~d} x}+\rho u^{2} \frac{\mathrm{d} u}{\mathrm{~d} x}-j E_{\perp}=0 \\
& h=\frac{\gamma}{\gamma-1} \frac{p}{\rho}
\end{aligned}
$$


where $\rho$ is density, $u$ is fluid velocity, $p$ is pressure, $j$ is current density, $B$ is magnetic field strength, $h$ is enthalpy, $\gamma$ is ratio of specific heat, $E_{\perp}$ is the transverse component of electric field.

The restriction imposed by Maxwell's equation, curl $\mathbf{E}=-\partial B / \partial t$, for a constant magnetic field and a one-dimensional problem require that be a constant, equal to $-V / w$ ( $V$ is voltage and $w$ is the distance between electrodes), throughout the channel. This constant can be expressed as some fraction of the entrance opencircuit field $u_{0} B$ as

$$
K=\frac{V}{u_{0} B w}
$$

where $K$ will be called the load parameter.

The generator is assumed to be segmented, and the segments are assumed to be infinitely thin, so that no axial currents flow. The proper Ohm's Law is

$$
j=\sigma\left(u B-\frac{V}{w}\right)
$$

where $\sigma$ is electrical conductivity includes Hall effects and ion slip, and $\mathbf{j}$ is parallel to $\mathbf{u} \times \mathbf{B}$. The restriction that $K$ is a constant places a restriction on the load resistance $R_{L}:\left(A_{e} j\right) R_{L}=$ $\left(A_{e j}\right)_{0} R_{L, 0}=$ constant

where $A_{e}$ is the electrode area, $R_{L}$ is load resistance, and the subscript zero denotes entrance values. If all electrodes are given the same area $A_{e}$, the current can be eliminated as follows:

$$
\frac{R_{L, 0}}{R_{L}}=\frac{j}{j_{0}}=\frac{\sigma\left(\frac{u}{u_{0}}-K\right)}{\sigma_{0}(1-K)}
$$

To solve the system of equations (28) to (31), the enthalpy $h$ can be eliminated by using equation (31) and the momentum and energy equations. The resulting expression can be integrated to obtain the following relation between the pressure and velocity:

$$
\begin{aligned}
& \left(\rho u^{2}+p\right)-\left(\rho_{0} u_{0}^{2}+p_{0}\right)=\frac{B w}{V} . \\
& {\left[\left(\frac{\gamma}{\gamma-1} u p+\frac{\rho u^{3}}{2}\right)-\left(\frac{\gamma}{\gamma-1} u_{0} p_{0}+\frac{\rho_{0} u_{0}^{3}}{2}\right)\right]}
\end{aligned}
$$

At this point, it is convenient to introduce the following non-dimensional variables and parameters:

$$
\begin{array}{ll}
U=\frac{u}{u_{0}}, \quad K_{L}=\frac{\gamma-1}{\gamma} K & , \quad P=\frac{p}{\rho_{0} u_{0}^{2}}, \\
M_{L}=1-\frac{2}{\gamma+1}\left(1-\frac{1}{M_{0}^{2}}\right), & M_{0}^{2}=\frac{\rho_{0} u_{0}^{2}}{p_{0}}, \\
\tau^{2}=\left(1-K_{L}\right)\left(M_{L}-K_{L}\right) &
\end{array}
$$

where $U$ is non-dimensional fluid velocity, $K_{L}$ is load voltage parameter, $P$ is non-dimensional pressure, $M_{L}$ is Mach number parameter, $M_{0}$ is entrance Mach number, $\tau$ is a parameter.

Equation (35) may then be expressed as

$$
\gamma P=U-\frac{\gamma+1}{2}\left(U-K_{L}-\frac{\tau^{2}}{U-K_{L}}\right)
$$

Equation (36) represents the relation between pressure and velocity. Since the duct is segmented with infinitely thin segments, the power developed in the generator can be obtained by integrating the product of voltage and current $V j H \mathrm{~d} x$ over the length of the generator:

$$
\Pi=\int_{0}^{L} V j H \mathrm{~d} x=\rho_{0} u_{0}^{3} K w H\left[\left(1+\frac{1}{\gamma M_{0}^{2}}\right)-(U+P)\right]
$$

where $H$ is the height of electrodes. 
This power can be compared to the total enthalpy flux entering the generator:

Total enthalpy flux $=\rho_{0} u_{0} w H\left(\frac{\gamma}{\gamma-1} \frac{p_{0}}{\rho_{0}}+\frac{1}{2} u_{0}^{2}\right)$

The ratio of these terms is called the conversion efficiency $\eta_{\text {conv }}$ and may be written as

$\eta_{c o n v}=\frac{K_{L}(1-U)}{\left(U-K_{L}\right)} \frac{\left(U-M_{L}\right)}{M_{L}}$

The power output of a generator with a specified inlet condition can now be determined. In order to calculate the output power density, however, a relation between velocity and generator length must be determined. The two variables, non-dimensional conductivity $\Omega=\sigma / \sigma_{0}$ and dimensionless interaction length $\xi$, defined by

$\xi=\frac{\sigma_{0} B^{2} x}{\rho_{0} u_{0}}$

are introduced. Equation (29) can then be written as

$\frac{\mathrm{d}}{\mathrm{d} \xi}(U+P)+\Omega(U-K)=0$

which can be expressed as

$$
\xi=\int_{U}^{1} \frac{1+\frac{\partial P}{\partial U}}{\Omega U(U-K)} \mathrm{d} U
$$

Equation (42) provides a relation between $U$ and the interaction length. An expression for $\partial P / \partial U$ can be obtained by differentiating equation (36):

$$
\frac{\partial P}{\partial U}=\frac{1}{\gamma}-\frac{\gamma+1}{2 \gamma}\left[1+\left(\frac{\tau}{U-K_{L}}\right)^{2}\right]
$$

so that equation (42) becomes $\xi=\frac{\gamma+1}{2 \gamma} \int_{U}^{1} \frac{1-\left(\frac{\tau}{U-K_{L}}\right)^{2}}{\Omega U(U-K)} \mathrm{d} U$

It is noticed that if the conductivity is constant $(\Omega=1)$, equation (44) can be integrated:

$$
\xi=\frac{\gamma+1}{2 \gamma}\left\{\begin{array}{l}
{\left[1-\left(\frac{\gamma \tau}{K}\right)^{2}\right] \ln \left(\frac{1-K}{U-K}\right)+} \\
+\left(\frac{\gamma \tau}{K}\right)^{2} \ln \left(\frac{1-K_{L}}{U-K_{L}}\right)- \\
-\left(\frac{\gamma \tau}{K}\right)^{2} \frac{K}{\gamma}\left(\frac{1}{1-K_{L}}-\frac{1}{U-K_{L}}\right)
\end{array}\right\}
$$

which is in agreement with the results of other investigations [8,9].

By using equation (44) for interaction length, it is possible to express the output power density $\wp$ as follows:

$\wp=\frac{\Pi}{w H L}=\sigma_{0} u_{0}^{2} B^{2} \frac{K_{L}(\gamma+1)(1-U)\left(U-M_{L}\right)}{2(\gamma-1)\left(U-K_{L}\right) \xi}$

This is the power density for a constant-area generator. It is of interest to gage the effect of velocity variation as well as conductivity variation. The power density at the entrance to the generator is

$$
\wp_{0}=\sigma_{0} u_{0}^{2} B^{2} K(1-K)
$$

The ratio of equation (46) to equation (47)

$$
\frac{\wp}{\wp_{0}}=\frac{(\gamma+1)(1-U)\left(U-M_{L}\right)}{2 \gamma \xi\left(U-K_{L}\right)(1-K)}
$$

will be used for comparison. This ratio will be calculated for the constant conductivity case, where $\xi_{\text {const }}$ is given by equation (45), and for $\xi$ as determined from equation (44) by use of the non-equilibrium conductivity.

The cycle thermodynamic efficiency may be conveniently expressed in terms of a generator

\section{Trang 42}


(isentropic) efficiency. This efficiency, which is defined as the actual change in total enthalpy of the working fluid in the generator compared to the change in total enthalpy for an isentropic process between the same total pressure conditions, is derived in section 2 above. The thermodynamic cycle efficiency for the Brayton cycle under conditions appropriate for space application is also calculated in section 2 . Certain limiting values for $\eta_{\text {conv }}$, however, can be obtained without specifying the conductivity.

\subsection{Limiting Case}

From equation (42) it can be seen that, as $U$ approaches $K, \quad \xi$ will approach infinity; obviously, this is a limiting value for $U$. This situation represents the maximum interaction length and, consequently, the maximum amount of energy that can be taken from the fluid. In some cases, however, the interaction length cannot become indefinitely large. It is limited by the phenomenon called "choking", which can be characterized by the criterion that the local Mach number reaches 1 . In the dimensionless symbols defined previously, this condition is equivalent to

$U=\gamma P$

This condition, when substituted into the equation (36), leads to the following specification of $U$ at choking:

$U_{c h}=K_{L}+\tau$

It is noticed that this is the value of the velocity for which the integrand in equation (44) is zero; that is, $U_{c h}$ is the condition that makes $\partial \xi / \partial \mathrm{U}=0$.

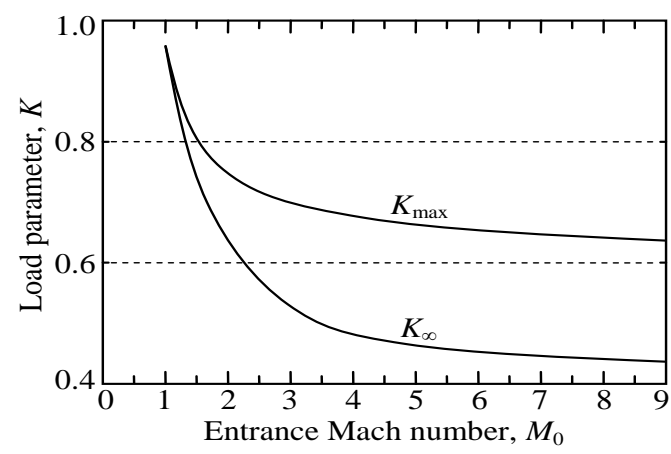

Figure 3. Load parameters $K$ (ratio of voltage to open-circuit voltage) for maximum thermal efficiency and infinite choking length for initial compressor efficiency of 0.8

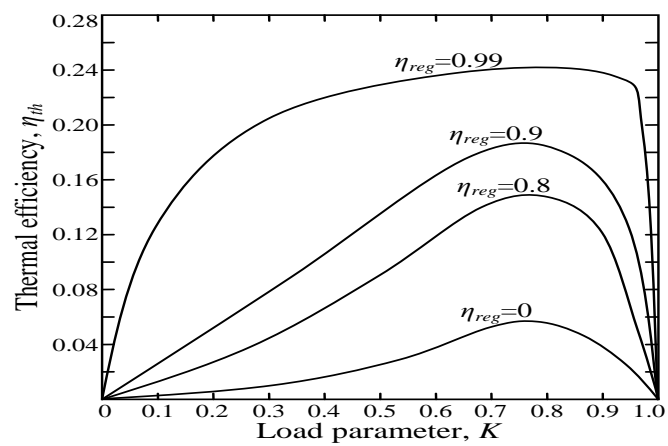

(a) Entrance Mach number of 2.0

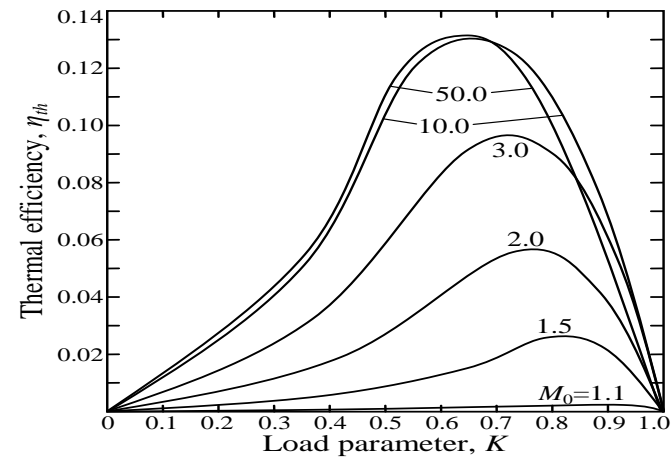

(b) Regenerator efficiency of 0.0 


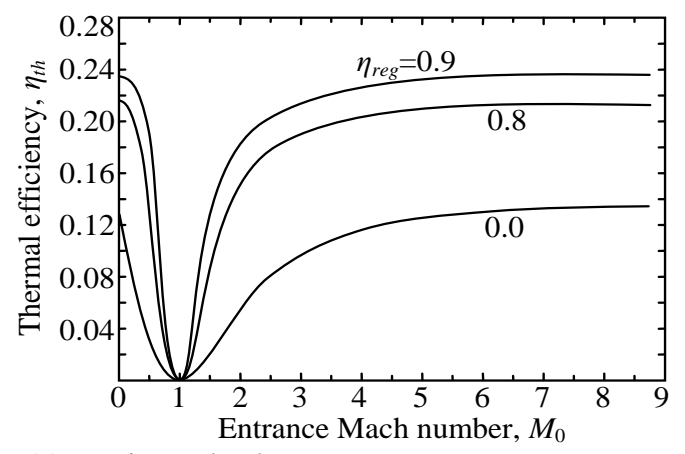

(c) Maximum load parameter

Figure 4. Thermal efficiency for limiting solution with compressor efficiency of 0.8

Two different operation limits have been described: first, when $U=K$ and the duct is infinitely long, and second, when $U=U_{c h}$ and the duct is choked. For any generator operation the proper limiting value can be determined by considering the case where the duct is choked at infinity. Formally, this occurs when $U_{c h}=K$. This condition can be substituted into equation (50) and the $K$ for which this occurs (call it $K_{\infty}$ ) can be determined from the following:

$$
K_{\infty}=\frac{\gamma-1}{\gamma} K_{\infty}+\tau
$$

which may be written as

$K_{\infty}=\gamma \frac{\sqrt{\frac{(\gamma-1)^{2}\left(1-M_{L}\right)^{2}}{4}+M_{L}}-\frac{(\gamma-1)\left(1+M_{L}\right)}{2}}{1-(\gamma-1)^{2}}$

The criterion for distinguishing between the two limiting cases may therefore be stated as follows: For $K>K_{\infty}$, the duct will not choke and $U$ will approach $K$, while for $K<K_{\infty}$, the duct will be choked and $U$ will approach $U_{c h}$. The duct is infinitely long and choked for $K=K_{\infty}$. When $\gamma=5 / 3, K_{\infty}$, is as shown in figure 3 . It is noted that for $M_{0}<1$ the duct will always choke, if sufficiently long, since $K$ must be less than 1; whereas, as shown in equation (52) $K_{\infty}$, must be greater than unity.
The quantity $\eta_{\text {conv }}$ can be calculated from equation (39) and $\eta_{S}$ from section 2 for a specified $\gamma$ and Mach number as a function of $K$. Therefore, the thermal efficiency $\eta_{\text {th }}$ can be calculated by means of equation (27) for a specified compressor efficiency and regenerator efficiency. In figure 4(a) this efficiency is plotted for $\gamma=5 / 3, M_{0}=2.0$, and $\eta_{\text {comp }}=0.8$ with regenerator efficiency as a parameter. Two items should be noted: first, the efficiency has a maximum at some values of $\mathrm{K}$, and second, this value of $K$ is independent of $\eta_{\text {reg }}$ even though the efficiency varies with $\eta_{\text {reg }}$ (this is true for all supersonic Mach numbers). The value of $K$ also depends on $\eta_{\text {comp }}$ but that dependency will not be investigated.

In figure 4(b), the efficiency is plotted again as a function of $K$ with $\gamma=5 / 3$ and $\eta_{\text {comp }}=0.8$, but with $\eta_{\text {reg }}=0$ and Mach number as the parameter. It can be seen that the $K$ for the optimum efficiency does depend on the Mach number. The value of $K$ for which the thermodynamic efficiency is optimized is called $K_{\max }$ and is shown in figure 3.

In figure 4(c), the efficiency at $K=K_{\max }$ and $\eta_{\text {comp }}=0.8$ is plotted as a function of Mach number with regenerator efficiency as a parameter. It can be seen that when $M_{0}>5$, the increases in thermal efficiency are insignificant. Therefore, there is no need for a high entrance Mach number more than 5 .

For the limiting values of $U, \eta_{c o n v}$ in equation (39) becomes

$$
\eta_{\text {conv }}=\frac{\gamma-1}{M_{L}}\left(1-K_{\max }\right)\left(K_{\max }-M_{L}\right) K>K_{\infty}
$$

or

$$
\eta_{\text {conv }}=\frac{K_{L}\left(1-U_{c h}\right)\left(U_{c h}-M_{L}\right)}{\left(U_{c h}-K_{L}\right) M_{L}} K<K_{\infty}
$$

\section{Trang 44}




\section{CONCLUSIONS}

In conclusion, it may be stated that a value of the load parameter which maximizes the thermodynamic efficiency of the limiting solution has been calculated. This value is independent of the regenerator efficiency, but dependent on Mach number, and the compressor efficiency (assumed to be 0.8 for all calculations presented herein).

For the limiting solutions the efficiency is independent of the form of the electrical conductivity. Of course, the electrical conductivity of the plasma is of great practical importance in that it largely determines the generator length required to extract power, which in turn determines the output power density of the generator. It is natural, then, to use the generator output power density as a means of comparing the usefulness of various working fluids (the larger the better, of course).

It is concluded that, if the duct is sufficiently long, for the entrance Mach numbers smaller than 1 , the duct will always choke. The thermal efficiency has a maximum at some values of load parameter, but this value of load parameter is independent of the regenerator efficiency even though the thermal efficiency varies with the regenerator efficiency. From the calculations, the load parameter for the optimum thermal efficiency clearly depends on the Mach number.

When the entrance Mach number is more than 5, the increases in thermal efficiency are insignificant. Therefore, there is no need for a high entrance Mach number.

The conductivity to be used in the calculation of output power density is that which is determined on the basis of the theory of magnetically induced ionization. This conductivity depends on the velocity as well as the usual parameters. All results obtained from this study will be much more significant for optimizing the efficiency of the MHD generator in the future works.

\section{Phân tích các đặc tính điện của máy phát từ thủy động để cực đại hiệu suất nhiệt}

\section{- Lê Chí Kiên}

Trường Đại học Sư phạm Kỹ thuật TP.HCM

\section{TÓM TÁT}

Bài báo này nghiên cứu máy phát Từ thủy động loại Faraday và xem xét ảnh hưởng của các thuộc tính điện đến hiệu suất nhiệt của hệ thống. Hoạt động của máy phát Từ thủy động được chỉ rõ bằng cách tối ưu hóa hiệu suất nhiệt có xét 
đến tham số tải và tối ưu hóa mật độ công suất phát ra có xét đến tỉ lệ chất cấy và áp suất làm việc. Theo kết quả phân tích, giá trị tham số tải mà làm cực đại hiệu suất nhiệt, không phụ thuộc vào hiệu suất bộ tái sinh nhiệt nhưng lại phụ thuộc vào số Mach và hiệu suất máy nén khí. Kết quả cũng cho thấy rằng không cần thiết số Mach ở cửa vào lớn hơn 5 vì khi đó hiệu suất nhiệt tăng không đáng kể.

Từ khóa: Máy phát MHD, hiệu suất nhiệt, đặc tính điện, tham số tải, mật độ công suất.

\section{TÀI LIẸU THAM KHẢO}

[1]. Na Zhang, Noam Lior, A novel Brayton cycle with the integration of liquid hydrogen cryogenic exergy utilization, International Journal of Hydrogen Energy, 33, 1, 214-224 (2008).

[2]. Emanuele Facchinetti, Martin Gassner, Matilde D'Amelio, François Marechal, Daniel Favrat, Process integration and optimization of a solid oxide fuel cell - Gas turbine hybrid cycle fueled with hydrothermally gasified waste biomass, Energy, 41, 1, 408-419 (2012).

[3]. Fredy Vélez, José J. Segovia, M. Carmen Martín, Gregorio Antolín, Farid Chejne, Ana Quijano, A technical, economical and market review of organic Rankine cycles for the conversion of low-grade heat for power generation, Renewable and Sustainable Energy Reviews, 16, 6, 41754189 (2012).

[4]. M. Bianchi, A. De Pascale, Bottoming cycles for electric energy generation: Parametric investigation of available and innovative solutions for the exploitation of low and medium temperature heat sources, Applied Energy, 88, 5, 1500-1509 (2011).
[5]. S.M. Aithal, Analysis of optimum power extraction in a MHD generator with spatially varying electrical conductivity, International Journal of Thermal Sciences, 47, 8, 1107-1112 (2008).

[6]. Naoyuki Kayukawa, Open-cycle magnetohydrodynamic electrical power generation: a review and future perspectives, Progress in Energy and Combustion Science, 30, 1, 33-60 (2004).

[7]. Moujin Zhang, S.T. John Yu, S.C. Henry Lin, Sin-Chung Chang, Isaiah Blankson, Solving the MHD equations by the spacetime conservation element and solution element method, Journal of Computational Physics, 214, 2, 599-617 (2006).

[8]. J. Reimann, L. Barleon, S. Dementjev, I. Platnieks, MHD-turbulence generation by cylinders in insulated ducts with different cross sections, Fusion Engineering and Design, 51-52, 49-854 (2000).

[9]. M. Turkyilmazoglu, Thermal radiation effects on the time-dependent MHD permeable flow having variable viscosity, International Journal of Thermal Sciences, 50, 1, 88-96 (2011). 